\title{
Les enjeux d'une approche anthropologique de la citoyenneté
}

Catherine Neveu

\section{(2) OpenEdition}

1 Journals

Édition électronique

URL : https://journals.openedition.org/remi/2024

DOI : $10.4000 /$ remi.2024

ISSN : $1777-5418$

Éditeur

Université de Poitiers

\section{Édition imprimée}

Date de publication : 1 décembre 2004

Pagination : 89-101

ISBN : 2-911627-38-5

ISSN : 0765-0752

Référence électronique

Catherine Neveu, «Les enjeux d'une approche anthropologique de la citoyenneté », Revue européenne des migrations internationales [En ligne], vol. 20 - $\mathrm{n}^{\circ} 3$ | 2004, mis en ligne le 25 septembre 2008, consulté le 14 avril 2022. URL : http://journals.openedition.org/remi/2024 ; DOI : https://doi.org/ 10.4000/remi.2024

Ce document a été généré automatiquement le 14 avril 2022.

(c) Université de Poitiers 


\title{
Les enjeux d'une approche anthropologique de la citoyenneté
}

\author{
Catherine Neveu
}

1 Souvent perçue comme une notion purement juridique, la citoyenneté peut de prime abord paraitre un objet bien improbable pour des anthropologues, et le faible nombre de travaux qui lui sont consacrés tendraient à confirmer cette première impression ${ }^{1}$. Effet d'un « grand partage » entre disciplines, polysémie et usage parfois immodéré de cette notion, échelle de mise en œuvre incongrue pour la discipline sont autant d'éléments pouvant expliquer le peu d'engouement que la citoyenneté semble y avoir suscité (Neveu, 1999). Le monde dans lequel nous vivons aujourd'hui est pourtant peuplé de citoyens; qu'elle soit définie comme un statut ou comme une capacité politique, la citoyenneté est en effet aujourd'hui une notion présente dans toutes les sociétés, quelles que soient les inégalités qui y persistent, les définitions des droits, formels ou réels, auxquels elle donne accès, ou les usages pratiques qui en sont faits.

2 Si ce «simple» constat ne constitue pas en soi un argument pour que les anthropologues s'y intéressent, de multiples dimensions de cet objet politique en font néanmoins un point d'entrée particulièrement pertinent pour qui veut comprendre et analyser un certain nombre d'enjeux centraux de notre époque. Il s'agit notamment des relations entre définitions juridiques ou normatives d'une part et représentations et pratiques sociales d'autre part; et des relations entre identité(s) et citoyenneté. Mais l'exploration de ces enjeux doit in fine être située par rapport à la manière même dont est définie la citoyenneté, statut ou capacité. S'interroger sur la citoyenneté, c'est donc nécessairement s'interroger sur la politique elle-même.

\section{La citoyenneté : un construit social et politique}

3 S'interroger d'un point de vue anthropologique sur la citoyenneté nécessite tout d'abord de se défaire de l'idée que cette notion n'aurait qu'une et une seule définition, qu'elle ne présenterait d'intérêt que philosophique ou normatif, ou encore qu'il ne s'agirait que d'un statut défini par l'État, restant relativement extérieur aux processus 
empiriquement observables dans des contextes locaux. Les approches classiques de la citoyenneté en sciences politiques la considèrent certes comme "un statut social (membership), codifié juridiquement et conférant un ensemble de droits aux individus à qui ce statut est reconnu. C'est aussi un ensemble d'obligations, formelles ou informelles, qui exigentle plus souvent - que les individus prennent part aux affaires de la Cité et participent activement aux affaires publiques d'une entité politique (l'État-Nation le plus souvent) dont ils sont membres " (Déloye, 2000). Quand bien même on se limiterait à une telle définition, aussi réductrice soit-elle, il resterait possible d'en faire un objet d'études anthropologiques, à la manière d'une anthropologie du droit (Rouland, 1991) ou d'une étude des modalités d'appropriation de ce statut par les groupes qui en sont détenteurs (voir ci-dessous). Il est toutefois nécessaire de dépasser cette définition purement juridique, pour inclure dans l'approche de la citoyenneté d'autres dimensions centrales de la notion. Leca (1991) précise ainsi que la citoyenneté soulève trois ordres de problèmes : celui des fondements de l'obligation politique; celui des conditions d'accès à la citoyenneté; et celui de la nature et de la portée des droits. Or, souligne-t-il aussitôt, ces conceptions sont "socialement et politiquement fabriquées", et les problèmes qu'elles soulèvent dépendent donc de la façon dont s'est constitué «le capital cognitif disponible dans une société ». Un tel caractère arbitraire (au sens de construit) est en effet aisément confirmé par un survol, même rapide, des évolutions dans la définition de telle ou telle « communauté citoyenne » au fil du temps ${ }^{2}$; ou par la variété des conceptions de la citoyenneté à l'œuvre dans différentes sociétés. Ainsi, l'importance qu'a revêtue la référence à la citoyenneté dans la société française, depuis la Révolution et l'instauration de la République, et l'insistance quasi-exclusive sur sa dimension politique, font du terme français "citoyenneté" un équivalent bien aléatoire de la "citizenship " anglaise ${ }^{3}$. Cette dernière est quant à elle profondément marquée par le modèle de Marshall, élaboré et conçu dans le cadre de la mise en place du «Welfare State » à l'issue de la Seconde Guerre mondiale 4 . Et celui-ci, pourtant érigé en modèle universel d'extension de la citoyenneté, «n'est que partiellement pertinent pour comprendre la citoyenneté en Espagne » (Garcia, 1994). En effet, contrairement à nombre de sociétés européennes, dans lesquelles l'État-providence se développa dès les années cinquante avec la participation de la société civile, l'expérience espagnole fut celle d'une politique d'assistance décidée par l'État franquiste sans la moindre participation de la dite société civile, les citoyens étant privés de leurs droits politiques élémentaires : «La notion de citoyenneté dans le cadre de l'État espagnol se trouve encore très conditionnée par l'expérience relativement récente de la dictature (...) Cela peut expliquer la résistance exprimée encore aujourd'hui à la notion d'éducation citoyenne » (Feixa-Pampols, 1985).

4 Non seulement donc les définitions et valeurs associées à la citoyenneté peuvent grandement varier d'une société à une autre, mais les conditions mêmes de leur élaboration influent sur l'appropriation dont elle peut faire l'objet de la part de citoyens eux-mêmes: "Venant de Grande-Bretagne puis du Canada, l'État-Providence incarnait pour moi un projet libéral progressiste; mais du point de vue des gens avec qui je travaillais dans cette partie de l'Espagne, il n'y avait jamais eu de prétention (convaincante) que l'État franquiste soit sur la voie de la construction de mécanismes institutionnels de plus en plus étendus visant leur participation en tant que citoyens (...) » (Smith, 1999). 


\section{Un objet ouvert à l'enquête empirique}

5 La notion de citoyenneté, les représentations dont elle fait l'objet, mais aussi les pratiques auxquelles elle donne lieu, offrent alors un champ particulièrement propice à une approche anthropologique. Ces représentations et pratiques incluent tout d'abord les multiples manières dont les institutions et les citoyens eux-mêmes construisent des représentations sociales quant aux droits et à leurs fondements, ainsi que sur la légitimité des uns et des autres (déjà inclus dans la collectivité légalement définie des citoyens ou exclus de celle-ci) à user de ces droits ou à y accéder. Quelques exemples tirés de recherches antérieures peuvent venir expliciter les enjeux d'analyse.

Dans un quartier londonien peuplé pour moitié de résidents originaires du Bangladesh, plusieurs processus ont pu être mis en lumière dans ces champs. S'agissant des droits et de leurs fondements, nombre de résidents "blancs» ne voyaient pas d'objection de fond à ce que leurs voisins bangladeshis disposent du droit de vote à toutes les élections ${ }^{5}$; au contraire, un certain nombre d'entre eux y voyaient une juste compensation des années de colonialisme. Par contre, ils soupçonnaient que les modalités d'usage de ce droit ne s'inscrivaient pas pleinement dans la logique purement politique qui aurait dû les inspirer. Ils considéraient ainsi que ces électeurs bangladeshis fondaient leur choix de vote, non pas sur des critères de programme politique ou d'appartenance partidaire, mais sur des affiliations villageoises ou de parenté ; autrement dit, ils opteraient plutôt pour un candidat lui-même d'origine bangladeshie parce qu'il venait du même village ou de la même région qu'eux. Outre qu'aucun élément empirique ne venait fonder cette représentation, elle était elle-même contradictoire avec la pratique de ces résidents «blancs ", qui avouaient voter euxmêmes pour tel ou tel candidat " parce qu'ils le connaissaient depuis longtemps ».

7 Dans ce même quartier, il était frappant de constater la force de l'attachement des résidents « blancs » au territoire local, leur insistance à la fois à en tracer des frontières très précises pour mieux le distinguer des quartiers environnants, et leur usage de ce "principe de localité » pour réclamer l'accès à certains droits, et notamment aux logements sociaux. À l'inverse, les résidents bangladeshis ne paraissaient pas faire montre du même attachement à l'espace local. Or en approfondissant l'analyse de ce qui pouvait paraitre relever, de prime abord, d'un «simple » processus d'identification locale différenciée selon l'origine, il devenait possible de réaliser à quel point celui-ci avait partie liée avec des représentations liées à la citoyenneté. Alors que les résidents «blancs » fondaient leurs revendications en matière de logement sur une implantation locale évaluée à l'aune du nombre de générations ayant vécu dans le quartier, la population bangladeshie revendiquait ce même accès en tant que citoyens britanniques, jouissant du même statut que leurs voisins «blancs » et devant donc avoir accès aux mêmes droits. Autrement dit, ce qu'ils recherchaient là était bien « une reconnaissance globale de la légitimité de leur présence et de leurs demandes, la fin de ce qu'ils perçoivent comme une situation de "citoyens de deuxième classe", une inscription positive et socialement reconnue dans un espace social et politique, celui de la société britannique » (Neveu, 1992).

8 On peut ainsi soumettre à l'enquête empirique non seulement les représentations dominantes, ou alternatives, concernant la légitimité de l'appartenance de telle ou telle fraction de la population à la "collectivité des citoyens", mais aussi les processus auxquels donnent lieu ces représentations. Ainsi, la société française fournit un 
exemple éclairant de la manière dont une définition strictement individualiste de la citoyenneté (le citoyen est d'abord et avant tout un individu, capable de s'extraire de ses affiliations particulières pour s'exprimer dans la sphère publique en en faisant abstraction) structure les représentations quant à la dite «intégration» des populations issues de l'immigration. Celle-ci est également conçue comme devant être purement individuelle, toute appartenance, réelle ou supposée, à d'autres collectivités (qualifiées de culturelles) étant perçue comme une dérive communautaire contraire à la définition purement politique de la citoyenneté. Le discours sur "l'intégration", (toujours soumise à ré-évaluation et dont l'achèvement peut indéfiniment être reporté) permet alors de maintenir ces populations dans une position d'extranéité par rapport à la «vraie» "communauté des citoyens », y compris en dépit de leur appartenance légale à celle-ci ${ }^{6}$. Cette extranéité construite s'accompagne alors d'une ethnicisation de ces populations, leurs prises de position et modes d'expression étant systématiquement analysés au filtre d'une appartenance ethnique, d'une "assignation identitaire ». De tels processus ont largement pu être observés par exemple dans une recherche menée il y a quelques années dans le Nord de la France; les jeunes Français d'origine algérienne étaient ainsi systématiquement sommés de "s'intégrer » (i.e. de faire la preuve de leur individuation et de leur détachement de toute logique " communautaire »), alors que dans le même temps, leurs prises de position, y compris lorsqu'elles s'inscrivaient explicitement dans le «modèle prescrit » d'une citoyenneté individualiste, étaient systématiquement analysées comme découlant de leur «ethnicité » (Neveu, 2001; 2003). On débouche alors sur ce que Balibar nomme «le paradoxe flagrant» de la politique française actuelle: "celui d'un "communautarisme républicain", qui fait de la non-reconnaissance culturelle, scolaire, administrative, des "identités particulières" (linguistiques, religieuses, nationales, etc.) au sein de la nation le critère de pureté auquel on reconnaîtrait son propre caractère d'universalité politique. Ainsi (...) la lutte contre les communautarismes plus ou moins réels dont on perçoit la menace se transforme en construction d'une identité exclusive qui, pour se définir de façon "abstraite" et "politique" (en particulier par la sacralisation de l'État, des institutions et des symboles de l'histoire républicaine française), n'en est pas moins utilisée très concrètement, pour tracer des lignes de démarcation ethniques (puisqu'il y a le peuple de la république, avec son histoire, ses symboles et ses traditions... et les autres)»(Balibar, 2001 ; souligné par l'auteur).

9 L'exploration des liens, problématiques, entre citoyenneté et identité, nous permettra de revenir sur ces questions. Mais les exemples qui précèdent permettent cependant d'insister sur une dimension souvent sous-estimée de la citoyenneté, sa dimension horizontale, celle des relations entre les citoyens eux-mêmes, ou entre ceux-ci et ceux qui ne le sont pas. La citoyenneté est en effet bien plus qu'un fait objectif, elle est éminemment projective (Murard et al, 1995), et ce caractère projectif s'exprime également dans les rapports horizontaux de concitoyenneté. C'est ce que Poche nomme "la citoyenneté comme reconnaissance", qui «met en jeu la façon dont la société se représente elle-même, c'est-à-dire se présente à elle-même, de façon réflexive, une image dans laquelle elle a choisi de se reconnaître ». Par ces processus de reconnaissance, «l'individu est reconnu "malgré" (malgré l'adoption par ailleurs d'autres références) (...) ou n'est pas reconnu "bien que" (bien qu'il fasse valoir des signes de reconnaissance, mais ils sont usurpés, ou ne convainquent personne, etc.) (...) » (Poche, 1992). Être citoyen, c'est en effet non seulement être dans une relation avec un État, mais c'est aussi être membre d'une collectivité, à la fois juridiquement constituée, et socialement construite. Plus encore, la question de l'appartenance à la collectivité des citoyens excède la délimitation proposée par les 
critères « objectifs » d'inclusion dans et d'exclusion de celle-ci ; on l'a vu à propos des processus sociaux permettant d'inclure ou d'exclure, au-delà du statut légal, telle ou telle fraction de la population dans cette collectivité, mais c'est d'autant plus vrai que ce sont les litiges sur les «conditions d'appartenance» qui fondent la citoyenneté (Rancière, 2000).

10 Avant (ou afin) de revenir sur cette dernière proposition, il faut tout d'abord explorer une autre facette de la citoyenneté, qui en fait un objet à la fois problématique et central pour l'anthropologie, celle de ses relations avec les identités.

\section{Citoyenneté(s) et identité(s)}

11 En fait, la question des relations entre citoyenneté et identité est le plus fréquemment posée à propos de l'identité nationale, à tel point que nationalité et citoyenneté sont fréquemment confondues. Cela n'a rien d'étonnant dans la mesure où la naissance de la « citoyenneté moderne » a correspondu avec la constitution de l'État-nation, et du fait qu'aujourd'hui encore, la plupart des droits liés au statut de citoyen sont attribués et pris en charge par lui. Il est pourtant indispensable, dans une époque où tend à s'imposer l'idée qu'à tout État doit correspondre une "ethnie » et une seule, que citoyenneté et nationalité ne sont pas équivalentes. Tout d'abord parce qu'il s'agit de deux statuts de nature différente, et qu'aucune loi internationale ou théorie de la citoyenneté ne construit cette équivalence; chaque État est libre de définir sa propre " collectivité des citoyens », et il n'y a aucune nécessité logique pour que celle-ci soit co-extensive avec la collectivité des nationaux. Ainsi à travers l'espace et le temps, des ressortissants d'un État ${ }^{7}$ ont pu être ou ne pas êtres citoyens : en France, jusqu'en 1947 les femmes étaient des «nationaux » tout en étant privées de droits essentiels, comme le droit de vote; en Grande-Bretagne, tout comme dans d'autres pays européens, la nationalité n'est pas une condition requise pour accéder aux droits politiques, ou tout du moins à certains d'entre eux.

12 Le débat tend à se complexifier quand, à la définition de la nationalité comme lien d'allégeance à un État, vient s'ajouter la perception de la nationalité comme identité nationale, comme sentiment d'appartenance, individuel ou collectif, à une collectivité partageant une histoire, une culture, une langue, etc. Il pourrait paraître préférable, ce qui n'est pas le cas en français, de disposer ici de deux termes différents, en nommant " étaticité » la première signification (Lochak, 1988), et "nationité » la seconde, en suivant ici Anderson (1983) quand il parle de nationness. Il pourrait alors être plus aisé d'éviter les confusions entre les deux notions de nationalité et de citoyenneté. En considérant la «nationité» comme l'identité culturelle du groupe dominant (numériquement et/ou politiquement), on pourrait ne la considérer que comme une seulement des multiples identifications disponibles pour les citoyens, ni plus ni moins légitime que d'autres. Mais dans bien des cas, cette "nationité », transfigurée par son ancrage dans la construction de l'État-nation, tend à être présentée comme le seul support d'un accès politiquement et socialement reconnu comme légitime aux droits découlant du statut de citoyen ${ }^{8}$.

13 Si donc il n'y a pas de fondements juridiques ou logiques à la confusion entre nationalité et citoyenneté, il y a par contre lieu de s'attacher à saisir quelles sont les configurations historiques, sociales et politiques qui peuvent déboucher soit sur une confusion, ou au contraire une relative dissociation, entre ces deux sphères, soit sur 
l'affaiblissement des autres formes d'identifications au bénéfice de la seule identité nationale. Certains travaux en France, comme ceux de Duchesne, ont ainsi pu y conclure à la disparition pure et simple des "autres appartenances naturelles (sic)", «la pluralité des groupes d'appartenance potentiels parmi lesquels on pourrait choisir" n'étant selon elle pas pensée par les citoyens qu'elle a interrogés, l'appartenance nationale leur paraissant la seule possible (Duchesne, 1994). À l'inverse, nombre de travaux d'anthropologie ont cherché à mettre en évidence, non pas la conformité à un modèle pré-établi de citoyenneté et de "nationité ", mais la complexité des articulations possibles entre ces différentes sphères d'appartenance ${ }^{9}$. Ainsi, si le projet d'une anthropologie politique contemporaine doit s'attacher à comprendre, comme le suggère Smith (1999), comment sont articulées, par les agents sociaux eux-mêmes, dans le cadre des États, appartenances à des collectivités locales et inscription dans un ensemble global, il est également nécessaire d'inclure dans ce travail l'articulation entre représentations de l'appartenance nationale véhiculées ou promues par l'État et représentations d'autres appartenances localisées; c'est par exemple ce qu'explore, de manière extrêmement stimulante, Herzfeld avec le concept d'« intimité culturelle» (2001).

\section{La citoyenneté, une « identité » parmi d'autres ?}

Doit-on pour autant considérer que la citoyenneté ne serait qu'une «identité » parmi d'autres, une sphère supplémentaire, mais de même nature que d'autres appartenances (sociales, culturelles...), les individus pouvant ainsi passer, au gré des contraintes, des moments ou de leurs stratégies, de leur "identité » en tant que citoyen à leur « identité » en tant que paysan ou que membre de telle ou telle entité culturelle ? Nul doute que chacun d'entre nous ne "passe " de l'une à l'autre de ses sphères, et ne se sente pas citoyen tout le temps et en toute circonstance. Mais nul doute également que d'une part on ne puisse si aisément dissocier l'appartenance culturelle et l'appartenance civique (Smith, 1999), mais que d'autre part il soit nécessaire de les distinguer. Quand il se propose de dégager les «traits culturels fondamentaux » de la citoyenneté, ceux en deçà desquels la notion elle-même n'existe plus, et ce abstraction faite des «codes culturels » de chaque société, Leca (1991) inscrit parmi ces traits fondamentaux «la séparation entre l'appartenance citoyenne et l'appartenance aux groupes sociaux auxquels on adhère plus immédiatement $d u$ fait de la prescription des rôles qu'ils imposent ». Cette séparation est au fondement de la citoyenneté dans la mesure où elle est ce qui permettrait à l'individu, dans la sphère publique, de s'exprimer en tant que citoyen, abstraction faite de tout autre appartenance (sociale, culturelle, religieuse, etc.). Du même coup, la citoyenneté ne serait pas "une identité parmi d'autres", mais précisément ce qui libèrerait l'individu de ses appartenances plus ou moins prescrites : "(elle) concerne ce refus, ce droit de dire non, d'entrer et de sortir des identités sans être piégé en elles. En ce sens, la citoyenneté est un rôle spécial, et non juste une identité parmi d'autres. C'est comme si elle remplissait une fonction de liaison (overarching) et de médiation dans le trafic des identités » (Van Gusteren, 1993).

C'est également ce que soutiennent d'ailleurs, à propos de la citoyenneté européenne, les tenants d'une citoyenneté "post-nationale ». Pour eux, la construction de l'Europe comme espace politique constitue une opportunité historique unique d'enfin dissocier citoyenneté et identité, et notamment identité nationale, et de redonner pleinement à 
la première sa dimension politique. Ainsi Tassin (1994) considère qu'il ne faut pas confondre "deux modes ou deux régimes de subjectivation individuelle", celui de l'identité, individuelle ou collective, qui peut trouver réponse dans le cadre de la nation; et celui de la citoyenneté, question politique qui «n'est pas celle de l'identité communautaire mais celle de l'activité publique $»^{10}$. Pour lui, «s'il appartient à la nation d'asseoir l'appartenance communautaire des individus sur une identification personnelle à des valeurs partagées, il appartient en revanche à l'espace public, espace civique et constitutionnel, de garantir non pas l'identité nationale de chacun et de tous, mais l'exercice des droits civiques et politiques qui donnent son sens politique aux actions des citoyens " (idem). Autrement dit, il s'agit de favoriser "la désunion de la référence politique et de l'appartenance culturelle» (Ferry, 1990). Or pour un certain nombre d'analystes, une telle désunion semble strictement impossible. Ainsi selon Schnapper, les tenants d'une citoyenneté post-nationale «tendent à sous-estimer non seulement les réalités ethniques de toute société réelle, mais surtout la nécessité d'intégrer ces réalités ethniques dans l'organisation politique concrète, y compris dans celles se réclamant du principe de citoyenneté » (Schnapper, 1997). Elle poursuit alors en rappelant que la Nation n'est pas qu'un projet civique basé sur l'abstraction de la citoyenneté: "La participation dans une société nationale est concrètement fondée sur toutes sortes d'éléments que l'on peut appeler ethniques : l'usage d'une langue commune (...) une culture commune et une mémoire historique particulière partagée par tous les nationaux, la participation aux mêmes institutions " (idem). Ce n'est pas le moindre des paradoxes que de voir une auteure défendant farouchement une vision strictement politique de la citoyenneté républicaine qualifier d'ethniques les références culturelles majoritaires. Mais cette argumentation a au moins le mérite d'une part d'attirer l'attention sur le poids des « capitaux cognitifs disponibles » dans une société donnée ; en rappelant le poids des « réalités ethniques dans l'organisation politique concrète, $\mathrm{y}$ compris dans celles se réclamant du principe de citoyenneté", Schnapper vient conforter la piste évoquée plus haut concernant la nécessité de s'attacher à saisir comment se sont constituées, et autour de quelles références, les conceptions de la citoyenneté à l'œuvre dans les différentes sociétés. Mais d'autre part, ce type d'analyse, considérant comme impossible une citoyenneté européenne déliée des références identitaires, doit nous pousser, comme le suggère Meehan, à mettre en cause un implicite prégnant, celui «selon lequel pour réussir, la politique doit être communautariste » (Meehan, 1996).

\section{Échelles de la citoyenneté}

16 Si la perspective européenne fournit aujourd'hui une occasion de réfléchir à nouveau frais sur les relations entre identité (nationale) et citoyenneté, il ne faudrait en effet pas penser qu'il ne s'agirait là que d'une question d'échelle. Le développement politique de l'Europe n'est pas tant l'occasion d'inventer un nouveau modèle de citoyenneté, qu'un moment spécifique de confrontation entre plusieurs conceptions de la citoyenneté, conceptions qui pré-existaient à cet épisode lui-même. En effet, la question soulevée à propos de la citoyenneté européenne est celle, déjà posée quand la citoyenneté se déploie dans l'espace d'un État-nation, des relations, nécessaires ou non, entre citoyenneté et identité. L'enjeu politique de la citoyenneté est-il "d'homogénéiser la culture des citoyens d'un État-Nation (...) et, de la sorte, délimiter l'espace de l'identité civique et nationale»(Déloye, 2000) ou de développer une 
« capacité d'exposer un litige et de reformuler les questions du droit et du non-droit » (Rancière, 2000) ?

Prendre au sérieux cette interrogation fondamentale sur l'enjeu même de la citoyenneté, ouvre alors l'exploration d'autres champs pour l'anthropologie, et c'est sur ceux-ci que l'on concluera cette réflexion. Certains des champs possibles de saisie des processus de subjectivation politique, comme capacité à « reformuler les questions du droit et du non-droit » ont déjà été évoqués plus haut ; c'est le cas par exemple des recherches qui tentent de saisir les multiples manières dont des individus ou des groupes élaborent leurs propres conceptions des droits auxquels eux ou d'autres devraient avoir accès, et les argumentaires avancés pour légitimer cet accès. Mais on peut étendre cette exploration à d'autres processus, à travers lesquels des citoyens ${ }^{11}$ s'emparent de la «chose publique » pour s'y affirmer en tant que sujets politiques. Si l'on en revient pour quelques temps à l'expérience française, mais ce constat est possible dans beaucoup d'autres sociétés contemporaines, on peut suggérer que l'ensemble des débats et des initiatives concernant le développement de formes plus participatives de démocratie fournissent de fertiles terrains de recherche. Ils permettent tout d'abord d'éprouver les modèles, explicites ou implicites, de démocratie (et donc de citoyenneté) à l'œuvre ; mais ils permettent également, au-delà de toute visée normative, de saisir empiriquement les multiples manières de s'éprouver en tant que citoyens, y compris à une autre échelle que celle de l'État, puisque nombre de ces initiatives s'inscrivent dans l'espace local d'une ville ou d'un quartier. On ne donnera ici que quelques exemples issus de recherches récentes ou en cours. À Roubaix, dans le Nord de la France, des Comités de quartier existent depuis plus de vingt ans; fondés à l'origine sur l'idée qu'il était nécessaire que les habitants participent plus activement aux prises de décision locales, ils se sont trouvé progressivement pris dans les contraintes et les injonctions liées à la mise en œuvre de nombreuses politiques publiques, notamment de "lutte contre l'exclusion». Sans qu'il soit possible ici de retracer en détail l'évolution de ces Comités et de leurs membres (pour plus de détails, voir Neveu, 2003), on peut toutefois souligner que cette analyse, et l'observation attentive des représentations et des pratiques à l'œuvre dans leur sein, a permis de mettre en lumière un système complexe de relations entre ces groupes militants et les pouvoirs publics. Système dans lequel un des enjeux centraux concernait la capacité de ces citoyens à disposer de (à se constituer) des "espaces publics partiels", dans lesquels ils pourraient définir eux-mêmes les questions devant faire l'objet de débat et les modalités de définition des "problèmes " à traiter. Or la question de l'autonomie (relative) de tels espaces publics partiels émergeait comme une réponse à un mode de fonctionnement des pouvoirs publics locaux basé assez largement sur une soumission complète de l'action collective aux injonctions, aux temporalités et aux nécessités des politiques publiques.

Une autre recherche, en cours, sur des instances de «démocratie de proximité » dans une autre ville française, permet de son côté d'explorer toute une série d'enjeux similaires. La promotion d'une démocratie plus participative, plus "proche des citoyens ", est aujourd'hui un leitmotiv de la vie politique française; une loi votée en février 2002 fait même obligation aux maires des communes de plus de 80000 habitants de mettre en place des instances de concertation ou de participation. Les modalités de désignation des citoyens associés à ces instances varient grandement d'une commune à l'autre: tirage au sort (sur les listes électorales, celles des abonnés au service de 
l'électricité, ou encore parmi des volontaires), élection, désignation parmi des personnalités associatives locales... Dans la grande majorité des cas, on peut cependant constater la mise en avant, implicite ou explicite, d'une certaine «norme démocratique », basée sur la primauté de la négociation et du consensus comme mode de régulation politique. L'observation des multiples manières qu'ont des citoyens " ordinaires" de s'approprier ces instances, d'y construire des rôles politiques différents, de tenter par leur biais d'influer sur les décisions politiques, mais aussi l'observation des résistances à cette "norme", offrent alors autant de supports à la mise en œuvre d'un regard anthropologique sur la citoyenneté.

De telles recherches offrent également l'intérêt d'aborder cet objet à l'échelle de la ville, et non plus de l'État, et de mettre concrètement en lumière les enjeux de relations entre identités « localisées » et civiques évoqués plus haut. Elles ouvrent également le champ des relations entre citoyenneté et territoire ${ }^{12}$, à travers l'analyse des constructions territoriales auxquelles donnent lieu ces instances participatives.

Une approche anthropologique de la citoyenneté nécessite donc de porter un regard critique tant sur les définitions normatives de la citoyenneté, que sur celles qui la réduisent à un statut ou en excluent les "rapports de citoyenneté ", c'est à dire les relations entre les citoyens eux-mêmes. Elle repose donc largement sur la mise en œuvre d'une réelle réflexivité de la part des anthropologues eux-mêmes, afin notamment de ne pas prendre notre propre imprégnation "nationale» pour un universel : " nous devons être très attentif à ne pas imposer notre propre définition particulière de l'État moderne et du rôle des citoyens en son sein, sur tout autre État moderne où il s'avère que nous menons un travail ethnographique » (Smith, 1999). Une telle réflexivité a déjà permis aux anthropologues de s'attacher à saisir « les lieux du politique » (Abélès, 1983) dans des sociétés fondées sur d'autres formes politiques que l'État ; elle est encore plus indispensable dans l'analyse du politique dans nos propres sociétés, sur un objet cristallisant les visions normatives, qu'il s'agisse de la dénégation de la conflictualité sociale ou politique, de la réduction du politique au rapport à l'État, ou de la confusion entre identité et politique.

\section{BIBLIOGRAPHIE}

ABÉLÈS Marc (1983) Le lieu du politique, Paris, Société d'ethnographie.

ANDERSON Benedict (1983) Imagined Communities. Reflections on the Origin and Spread of Nationalism, Londres, Verso.

BALIBAR Étienne (2001) Une citoyenneté sans communauté ?, in Étienne Balibar Éd., Nous, citoyens d'Europe ? Les frontières, l'Etat, le peuple, Paris, La Découverte.

DÉLOYE Yves (2000) Explorer le concept de citoyenneté européenne : une approche sociohistorique, Yearbook or European Studies, volume XIV.

DUCHESNE Sophie (1994) Citoyenneté à la française, Paris, Presses de Sciences Po. 
DUMONT Louis (1991) L'idéologie allemande. France-Allemagne et retour, Paris, Gallimard.

FEIXA-PAMPOL C. (1998) Identités sociales, identités citoyennes en Espagne et en Catalogne : du franquisme à nos jours, Agora Débats Jeunesses, 12.

FERRY Jean-Marc (1990) Qu'est-ce qu'une identité postnationale ?, Esprit, nº 164.

GARCIA S. (1994) The Spanish experience and its implication for a Citizen's Europe, in V.A. Goddard, J.R. Llobera et C. Shore Dirs., The Anthropology of Europe, Identities and Boundaries in Conflict, Oxford, Berg.

HERZFELD Michael (1997) Cultural Intimacy. Social Poetics in the Nation-State, Londres, Routledge.

LECA Jean (1991) Individualisme et citoyenneté, in P. Birnbaum et J. Leca Dirs., Sur l'individualisme, Paris, Presses de Sciences Po.

LOCHAK Danièle (1988) Étranger et citoyen au regard du droit, in C. Withol de WENDEN Dir., La citoyenneté, Paris, Edilig-Fondation Diderot.

MEEHAN Élisabeth (1996) European integration and citizens' rights : a comparative perspective, Publius, volume XXVI, $\mathrm{n}^{\circ} 4$.

MURARD Numa Dir., (1995) Pauvres et citoyens? Faites vos preuves! Rapport de recherche au FAS, Paris, Association Trass.

NEVEU Catherine (1992) Espace et territoire à Spitalfields : perceptions locales et pratiques municipales, Espaces et Sociétés, $\mathrm{n}^{\circ}$ 68/1.

NEVEU Catherine (1993), Communauté, nationalité, citoyenneté. De l'autre côté du miroir, les Bangladeshis de Londres, Paris, Editions Karthala.

NEVEU Catherine (1997) Anthropologie de la citoyenneté, in M. Abélès et H-P. Jeudy Dirs., Anthropologie du politique, Paris, Armand Colin.

NEVEU Catherine (2001) L'accès à l'espace public entre politiques publiques et « dérive ethnique ». L'expérience d'associations dites « de jeunes » à Roubaix, in A. Vulbeau Dir., La jeunesse dans l'espace public : expérimentation et expériences, Cahiers interdisciplinaires de recherche sur les banlieues.

NEVEU Catherine (2003) Citoyens, habitants et jeunes. Citoyenneté et espace public dans une ville du Nord, à paraître, Presses Universitaires du Septentrion.

POCHE Bernard (1992) Citoyenneté et représentation de l'appartenance, Espaces et Sociétés, ${ }^{\circ}$ $68 / 1$.

RANCIÈRE Jacques (2000) Citoyenneté, culture et politique, in M. Elbaz et D. Helly Dirs., Mondialisation, citoyenneté et multiculturalisme, Paris, L'Harmattan / Presses Universitaires de Laval.

ROULAND Norbert (1991) Aux confins du droit. Anthropologie juridique de la modernité, Paris, Odile Jacob.

SCHNAPPER Dominique (1997) The European debate on citizenship, Daedalus, Volume 126, $\mathrm{n}^{\circ} 3$.

SMITH Gavin (1999) Confronting the present. Towards a politically engaged anthropology, Oxford, Berg.

TASSIN Étienne (1994) Identités nationales et citoyenneté politique, Esprit, janvier.

VAN GUSTEREN Hermann (1993) Contemporary citizenship and plurality, contribution au workshop « Citizenship and Plurality », European Consortium for Political Research, Leiden, avril. 


\section{NOTES}

1. Cet article a fait l'objet d'une publication en bulgare dans Sotziologitzeski problemi, 2/2003 automne-hiver

2. On peut ainsi évoquer l'accès, assez récent en France, des femmes au droit de vote, ou l'abaissement de l'âge de la majorité légale de 18 à 21 ans.

3. Dumont considère ainsi «/.../ que le Français se sent tel essentiellement comme citoyen. La France, c'est pour lui avant tout la démocratie, la république /.../ Telle est la forme que prend chez nous l'ethnocentrisme, disons plutôt sociocentrisme, que l'on rencontre dans toute société qui porte à poser une différence fondamentale en valeur, entre "nous" et "les autres" " (Dumont, 1991).

4. Marshall a élaboré une théorie basée sur la mise en évidence d'une séquence d'extension des droits du citoyen, des droits civils aux droits politiques, puis aux droits sociaux (Marshall, T.H., (1963) Class, citizenship and social development). Pour une critique des fondements idéologiques de cette théorie, voir entre autres Smith, 1999.

5. À l'époque de cette recherche (fin des années 1980), les résidents légaux originaires du Commonwealth disposaient, en tant que sujets de sa majesté, du droit de vote aux élections locales et générales, quelle que soit leur nationalité. Voir Neveu, 1993.

6. Processus qui renvoie à ce que Poche appelle "le champ de la reconnaissance », voir cidessous.

7. Au sens d'individus qui sont dans une relation d'allégeance avec un Etat. On dirait « nationaux " en français, et « citizens » en anglais, ce qui montre assez à quel point les confusions sont possibles entre ces deux notions et ce qu'elles recouvrent.

8. De ce point de vue, et de manière paradoxale, l'opposition classique entre un modèle de la " nation culturelle » à l'allemande, et un modèle de l'« État-nation » politique à la française perd de sa force, dans la mesure où y compris le projet d'une « nation politique " peut en arriver à être naturalisé, les « compétences » requises pour s'y conformer finissant par être construites comme l'apanage des seuls citoyens « de souche». Voir à ce sujet entre autres Balibar, 2001.

9. Pour ne s'en tenir qu'au cas français, voir entre autres W. Lem (1999) Cultivating dissent : work and politics in Languedoc; M. McDonald (1989) We are not the French!

10. Ou comme le dit Rancière (2000) : «Les groupes d'appartenance, définis par une naissance, une histoire, une religion, une langue, voire même des idées et aspirations communes sont une chose. Les formes de la subjectivation politique en sont une autre. Celles-ci ne sont pas des communautés d'appartenance. Ce qui constitue le sujet politique (...) ce n'est pas l'expression d'une identité mais d'une hétérologie », et donc « La communauté politique n'est pas définie par le rassemblement de tous ceux qui ont la même appartenance. Elle est définie par l'ensemble des litiges sur l'appartenance. Elle est la communauté fondée sur le partage de ces litiges ».

11. Et donc ici toute personne mettant en œuvre ce type de capacité, et non les seuls nationaux ou citoyens de statut.

12. On retrouve ici une des pistes, essentielles, proposées par Poche, pour qui «la reconnaissance de la citoyenneté n'est pas seulement une acceptation avouée, mais immatérielle; elle est aussi partage du topos /.../ reconnaissance de la co-présence dans le territoire au quotidien /.../ (et) publicité reconnue au parcours du territoire auquel est attachée la citoyenneté » (Poche, 1992). 


\section{RÉSUMÉS}

$\mathrm{Au}$-delà des approches strictement juridiques la considérant comme un statut, la citoyenneté peut être analysée en tant qu'elle est un construit social et politique, et une activité, dont les représentations et les significations varient dans le temps et l'espace. À ce titre, elle peut faire l'objet d'approches empiriques multiples, concernant ses modalités d'appropriation et d'usage, que ce soit dans les relations entre différents groupes au sein d'une population, ou entre citoyens et institutions. La question des liens entre citoyenneté et identité, ainsi que celle des échelles de la citoyenneté, revêt alors une importance toute particulière.

For an Anthropological Approach to Citizenship. Beyond strictly juridical approaches considering it as a status, citizenship can be analyzed as a social and political construct, an activity, carrying a variety of meanings and representations through time and space. As such it can be studied through multiple empirical research, concerning how it is used and made sense of in relations between different groups, or between citizens and institutions. The links between citizenship and identity on the one hand, and the scales of citizenship on the other, thus constitute issues of particular relevance.

Las claves de una visión antropológica de la ciudadanía. Más allá de las visiones estrictamente jurídicas que identifican la ciudadanía como un estatus, esta ser analizada como una construcción social y política, como una actividad cuyas representaciones y significados varían en el tiempo y en el espacio. En este sentido, el análisis de la ciudadanía puede basarse en métodos empíricos múltiples destinados a explorar las modalidades de apropiación y de uso de la ciudadanía en las relaciones entre diferentes grupos, en el seno de una población o entre ciudadanos e instituciones. Dos cuestiones revisten, en este caso, una importancia particular : los lazos entre la ciudadanía y la identidad y las escalas de la ciudadanía.

\section{INDEX}

Mots-clés : citoyenneté, identité nationale, relations interethniques

\section{AUTEUR}

\section{CATHERINE NEVEU}

Laboratoire d'Anthropologie des Institutions et des Organisations Sociales (LAIOS), Paris 